\title{
Performance Analysis of Image Watermarking Using Contourlet Transform and Extraction Using Independent Component Analysis
}

\author{
S. Saju*, G. Thirugnanam \\ India. \\ * Corresponding author. Tel.: 91-9597594167; email: Sajube82@gmail.com \\ Manuscript submitted August 20, 2015; accepted October 24, 2015. \\ doi: 10.17706/jcp.11.3.258-265
}

Dept. of Electronics \& Instrumentation Engg., Annamalai University, Annamalai Nagar- 608002., Tamilnadu,

\begin{abstract}
In this paper, performance analysis of digital image watermarking using contourlet transform is proposed. The ease of digital media modification and dissemination necessitates content protection beyond encryption.So information's are hidden as digital watermarks in multimedia enables protection mechanism in decrypted contents. Among emergent applications of digital watermarking, owner identification, proof of ownership and transaction tracking are applications that protect data by embedding the owner's information in it.The main challenge in exploring geometry in images comes from the discrete nature of the data. Thus, unlike other approaches, our approach starts with a discrete-domain construction and then studies its convergence to an expansion in the continuous domain. Many literature have reported about Discrete Wavelet Transform watermarking techniques for data security. However, DWT based watermarking schemes are found to be less robust against image processing attacks and the shift variance of Wavelet Packet Transform causes inaccurate extraction. In Contourlet transformation, original image is decomposed to two level using contourlet and the watermark is embedded in the resultant sub-bands. Sub-band selection is based on the value of Peak Signal to Noise Ratio (PSNR) that is calculated between watermarked and original image.In DWT-SVD watermarking technique, firstly original image is decomposed according to DWT and then watermark is embedded in singular values obtained by applying SVD.To extract the watermark, ICA-ML is used, it has a novel characteristic is that it does not require the transformation process to extract the watermark. Simulation results show that contourlet based watermarking scheme is robust against attacks such as Salt and Pepper noise, Median filtering and rotation. The performance measures like PSNR and Similarity measure are evaluated and compared with SVD algorithm to prove the robustness of the scheme. Simulations are carried out using Matlab Software.
\end{abstract}

Key words: Watermarking, contourlet, SVD, independent component analysis.

\section{Introduction}

One of the most important advantages of the numeric era is the widespread use of Internet and computers, which is the result of exchanging digital media. However, illegal reproduction of data has also emerged with this extraordinary revolution and is raising questions and concerns about ownership rights. As a solution to this issue, we found Digital watermarking which consists in embedding digital data into digital contents in order to guarantee the ownership and the integrity. The basic requirements for a secure watermarking scheme are imperceptibility, robustness, capacity and security. Digital watermarking is the process by which a discrete data stream called a watermark is hidden within a host multimedia signal by 
imposing imperceptible changes on the signal. In many proposed techniques, this procedure entails the use of a secret key that must be used to successfully embed and extract the watermark. One major driving force for research in this area is the need for effective copyright protection scenarios for digital media. In such an application, a serial number or copy protection code is watermarked into the signal to protect ownership or user rights. It is expected that an attacker will attempt to remove the watermark by intentionally modifying the watermarked signal. Thus, we must strive to embed the mark such that it is difficult to remove (without the use of the key) unless the marked signal is significantly distorted. A popular analogy for watermarking is the process of data communications in which the goal is to effectively communicate the watermark information using information hiding techniques. Therefore, it is crucial for the future development of networked multimedia systems that robust methods are developed to protect the intellectual property right of data owners against unauthorized copying and redistribution of the material made available on the network. Furthermore, it is an important issue to develop a robust watermarking scheme with a better tradeoff between robustness and imperceptibility [1].

Watermarking techniques can be broadly classified into two categories: such as spatial domain methods and transform domain methods. Spatial domain methods are less complex as no transform is used, but are not robust against attacks. Transform domain watermarking techniques are more robust in comparison to spatial domain methods. This is due to the fact that when image is inverse wavelet transformed watermark is distributed irregularly over the image, making the attacker difficult to read or modify [2].The contourlet transform is a directional multi resolution expansion, which can represent images containing contours efficiently. Contourlet transform possess all features of wavelets and also shows a high degree of directionality and anisotropy. One of the unique properties of contourlet transform is that we could have any number of directional decompositions at every level of resolutions.

This paper is organized as follows: Section 2 reviews the DWT, Section 3 discuss contourlet transform, Section 4 pointed out hybrid SVD, in Section 5 watermark extraction technique ICA is explained. Simulation results are presented in Section 6 and conclusions are elaborated in Section 7.

\section{Discrete Wavelet Transform}

A two dimensional DWT is shown in Fig. 1, where $h[-m]$ and $g[-m]$ are the low pass and high pass analysis filters, while the corresponding low pass and high pass synthesis filters are $h[m]$ and $g[m] ; c_{j}$ and $d_{j}$ are the low band and high band output coefficients at level $\mathrm{j}$ [3].

DWT analysis is given by

$$
\begin{aligned}
& c_{j}[m, n]=\left\langle c_{j+1}(m, n) * h[-m]\right) \downarrow 2 \\
& d_{j}[m, n]=\left\langle c_{j+1}(m, n) * g[-m]\right) \downarrow 2
\end{aligned}
$$

Similarly DWT synthesis is given by

$$
c_{j+1}[m, n]=\left[\left(c_{j}(m, n) \uparrow 2\right) * h[m]+\left(d_{j}(m, n) \uparrow 2\right) * g[m]\right]
$$

where $*$ denotes convolution, $\downarrow 2$ and $\uparrow 2$ denotes downsampling and upsampling by a factor of two, $c_{j}$ denotes approximation coefficient and $d_{j}$ denotes detail coefficients.

\section{Contourlet Transform}

An image can be effectively represented using the following features: 
1) Multiresolution. The representation should allow images to be successively approximated, from coarse to fine resolutions.

2) Localization. The basis elements in the representation should be localized in both the spatial and the frequency domains.

3) Critical sampling. For some applications (e.g., compression), the representation should form a basis, or a frame with small redundancy.

4) Directionality. The representation should contain basis elements oriented at a variety of directions, much more than the few directions that are offered by separable wavelets.

5) Anisotropy. To capture smooth contours in images, the representation should contain basis elements using a variety of elongated shapes with different aspect ratios.

Among these requirements, the first three are successfully provided by separable wavelets, while the last two require new constructions. Moreover, a major challenge in capturing geometry and directionality in images comes from the discrete nature of the data.The input is typically sampled images defined on rectangular grids [3]. For example, directions other than horizontal and vertical look very different on a rectangular grid. Because of pixelization, the notions of smooth contours on sampled images are not obvious. For these reasons, unlike other transforms that were initially developed in the continuous domain and then discretized for sampled data.

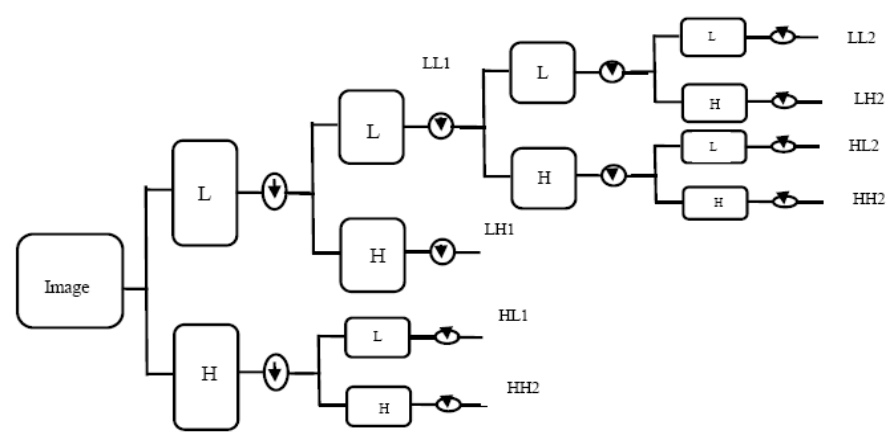

Fig. 1. A two dimensional decomposition using DWT.

Contourlet transform is a multi resolution and multidirectional transformation technique which is used in image analysis for capturing contours and fine details in images [2]. The contourlet transform is composed of basis functions oriented at different directions in multiple scales with flexible aspect ratios. This frame work should form a basis with small redundancy unlike other transform techniques in image processing, Contourlet representation contains basis elements oriented at variety of directions much more than few directions that are offered by other separable transform technique. One way to obtain a sparse expansion for images with smooth contours is first apply a multistage wavelet like transform to capture the edge points, and then local directional transform to gather the nearby edge points into contour segments.

With this insight, we can able to construct a double filter bank structure shown in Fig. 2 where the laplacian pyramidal (LP) filter is used to capture the point discontinuities, followed by a directional filter bank (DFB) to link point discontinuities into linear structures. The overall result is an image expansion using basic elements like contour segments, and thus it is named contourlet transform. The combination of this double filter bank is named pyramidal directional filter bank (PDFB). Fig. 2 shows a multiscale and directional decomposition using a combination of a LP and a DFB. Band pass images from the LP are fed into a DFB so that directional information can be captured.

The original image is decomposed into using low pass filter followed by Directional Filter Banks. The proposed watermark embedding scheme is shown in Fig. 3, the original image is passed through a low pass and high pass filter where the low pass output is decomposed using DFB. The resultant sub-bands are 
ordered as per low to high frequency components. The watermark is embedded in each of these Contourlet sub-bands and corresponding PSNR values are calculated. The embedded Contourlet coefficients are combined together using Inverse DFB. Once again passed through inverse wavelet low pass and high pass filters to obtain watermarked image.

$$
W_{C F}(i, j)=C F(i, j)+\alpha \cdot W_{i, j}
$$

where $W_{C F}(i, j)$ is the watermarked Contourlet coefficients (1 to 7$), C F(i, j)$ is the Contourlet coefficient (1 to 7), $W_{i, j}$ is the watermark and $\alpha$ denote the embedding factor.

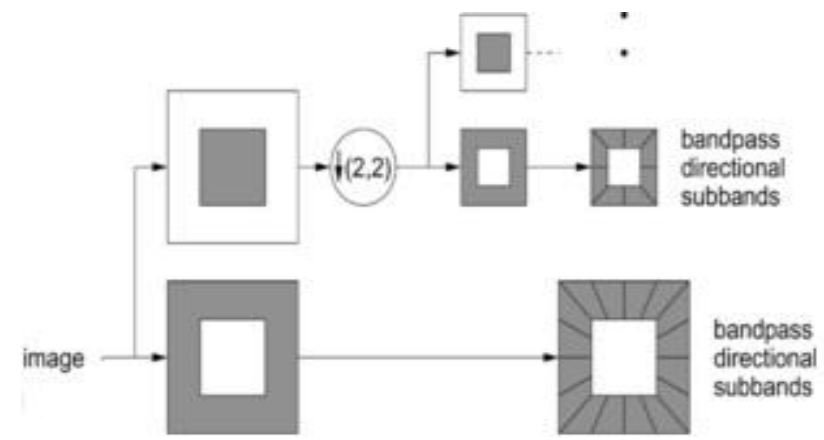

Fig. 2. Contourlet filter bank.

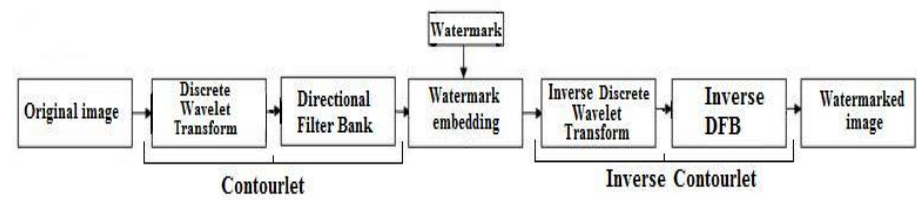

Fig. 3. Proposed watermark embedding scheme.

\section{Singular Value Decomposition}

Singular Value Decomposition is a linear algebra transform which is used for factorization of a real or complex matrix with numerous applications in various fields of image processing. As a digital image can be represented in a matrix form with its entries giving the intensity value of each pixel in the image, SVD of an image $M$ with dimensions $m \times m$ is given by,

$$
M=U S V-1
$$

Where, $U$ and $V$ are orthogonal matrices and $\mathrm{S}$ known as singular matrix is a diagonal matrix carrying non-negative singular values of matrix $M$. The columns of $U$ and $V$ are called left and right singular vectors of $M$, respectively. They basically specify the geometry details of the original image. Left singular matrix i.e., $U$ represents the horizontal details and right singular matrix i.e., $V$ represents the vertical details of the original image. The diagonal values of matrix $S$ are arranged in decreasing order which signifies that the importance of the entries is decreasing from first singular value to the last one.

There are two main properties of SVD to employ in digital watermarking schemes :

Small variations in singular values do not affect the quality of image.

Singular values of an image have high stability

Hybrid technique is a fusion of two techniques. Here, DWT and SVD are used together to improve the quality of digital watermarking and hence increases the robustness and imperceptibility of an image.

The embedding algorithm for DWT-SVD based watermarking is shown in Fig. 4. The algorithm works as :

Step 1: The original $N^{*} N$ Gray image is transformed into sub-bands using single level 2-D DWT. 
Step 2: SVD is performed on LL sub-band (on Gray components) of decomposed Gray original image

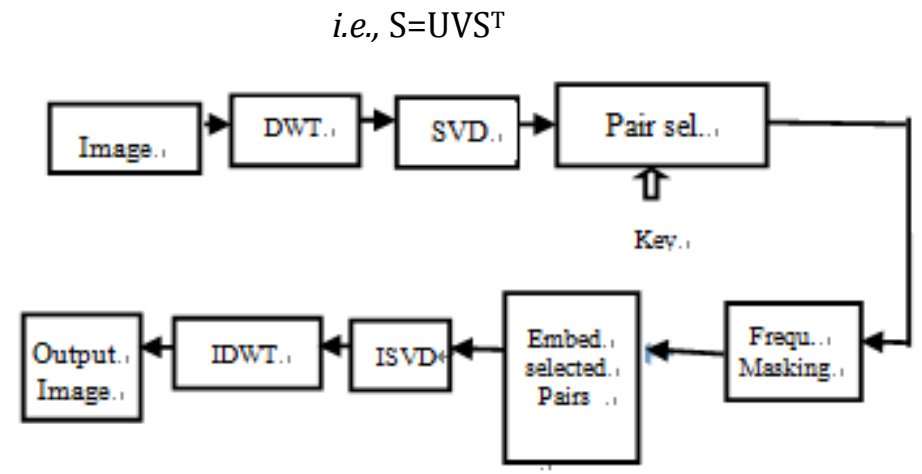

Fig. 4. SVD based embedding.

Step 3: The watermark of size $M^{*} M$ Gray image is transformed into sub-bands using single level 2-D DWT. Step 4: SVD is performed on LL sub-band (on Gray components) of decomposed Gray watermark image

$$
\text { i.e., } S W=U_{w} S_{w} V_{w}{ }^{T}
$$

Step 5: After performing SVD on both original and watermark images, the resultant watermark image is then embedded with the original image using the scale factor $(\alpha)$

$$
\text { i.e., } S W I=S+\alpha(S W)
$$

Step 6: Inverse SVD is performed on embedded image.

Step 7: Finally, inverse 2-D DWT is performed to produce the watermarked image.

\section{Independent Component Analysis}

This section briefly reviews ICA algorithm and how ICA is applied to watermark extraction. ICA aims at extracting unknown hidden components from multivariate data using only the assumption that the unknown factors are mutually independent.

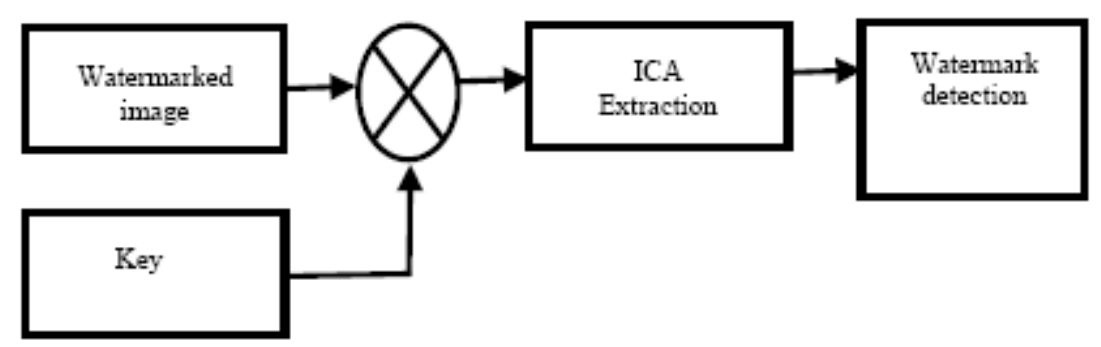

Fig. 5. ICA based extraction.

Independent component analysis (ICA) is a novel statistical technique that aims at finding linear projections of the data that maximize their mutual independence. ICA has received attention because of its potential applications in signal processing such as in feature extraction, and Blind Source Separation (BSS) with special emphasis to physiological data analysis and audio signal processing. The goal of BSS is to recover the source signals given only sensor observations that are linear mixtures of independent source signals. ICA is a statistical technique for obtaining independent sources, $S$ from their linear mixtures, $X$, when neither the original sources nor the actual mixing, $A$ are known.

\subsection{Maximum Likelihood ICA (ICA ML)}

The Maximum Likelihood ICA (ICA ML) method for estimating the optimal unmixing matrix W. ICA ML estimation is a standard statistical tool for finding parameter values (e.g., the unmixing matrix $\mathbf{W}$ ) that 
provide the best fit of some data (e.g., the signals $\hat{S}$ extracted by $\mathbf{W}$ ) to a given a model. The objective of ICA ML is to find an unmixing matrix $\mathbf{W}$ that yields extracted signals which is given by

$$
\hat{S}=W X,
$$

where $X$ is the mixing matrix. This model incorporates the assumptions that source signals are non-Gaussian and independent. ICA ML is outlined as follows:

(i) Centre the data to make its mean zero.

(ii) Choose an initial separating matrix $W$, initial values $\gamma_{i}, i=1, \ldots, n$ and learning rates $\mu$ and $\mu_{\gamma}$ randomly.

(iii) Compute $\hat{S}=W X$,

(iv) If the nonlinearities are not fixed. Then update

$$
\gamma_{i}^{+}=\left(1-\mu_{r}\right) \gamma_{i}+\mu_{r} E\left\{-\tanh \left(y_{i}\right) y_{i}+\left(1-\tanh \left(y_{i}\right)^{2}\right\}\right.
$$

If $\gamma_{i}^{+}>0$ define $g_{i}$ as

$g^{+}(y)=-2 \tanh (y)$, otherwise

$$
g^{-}(y)=\tan h(y)-y
$$

(v) Update the separating matrix is given by

$$
W^{+}=W+\mu\left[I+g(y) y^{T}\right] B
$$

where $g(y)=\left(g_{1}\left(y_{1}\right), \ldots, g_{n}\left(y_{n}\right)\right)^{T}$

(vi) If not converged, go back to step (iii)

To perform ICA ML, a linear mixture of image is generated to demix the watermark signal from the mixtures. The novelty of this ICA detector is that it does not require the transform process to separate the watermark and omits using original image. In this paper, watermark is embedded in Contourlet transform sub-bands and the location of embedding is the same in spatial domain. Therefore ICA is applied directly on the watermarked image.

The mixtures can be modeled as

$$
\begin{aligned}
& X_{1}=a_{11} C F_{1}+a_{12} W \\
& X_{2}=a_{21} C F_{2}+a_{22} W \\
& X_{3}=a_{31} C F_{3}+a_{32} W
\end{aligned}
$$

where $X_{1}, X_{2}, X_{3}$ are mixtures, $C F$ is the watermarked contourlet coefficients, $a$ is a mixing matrix, $W$ is the watermark matrix and $K$ is a random key in the embedding process. Applying the above mentioned ICA algorithms to those mixtures, matrix watermark $W$ is extracted. Similarity measure between original and extracted watermark is calculated using the formula given below:

$$
\operatorname{sim}\left(X, X^{\prime}\right)=\frac{X \cdot X^{\prime}}{\sqrt{X^{\prime} \cdot X^{\prime}}}
$$

where $X$ - Original watermark, $X^{\prime}$ - Extracted watermark. 


\section{Simulation Results}

The proposed watermarking scheme is tested on Barbara image of size $256 \times 256$. Two level DWT is followed by DFB is performed on the original image. The frequency sub-bands are selected to embed watermark as explained in Section 3. A Hand image of size $64 \times 64$ is used as watermark and $\alpha$ is set to 0.3 by repeated simulation to ensure the invisibility of the watermark. Fig. 6 shows the Original image, Fig. 7 and Fig. 8 show the one and two level decompositions. Fig. 9 shows the Contourlet coefficients using DFB and Fig. 10 show the watermark. Watermark is embedded in all sub-bands of Contourlet transform and their corresponding values are tabulated in Table 1. From the results, it is inferred that watermark embedded in 7th sub-bands generates more PSNR when compared to other sub-bands. From the results shown it is inferred that, both original and watermarked images are evidently indistinguishable and imperceptible. The robustness of the above watermarking scheme is validated against various attacks like Salt \& Pepper noise, Median Filtering and Rotation are shown in Fig. 12-Fig. 14 respectively. Table 1 also compares the values of PSNR and Similarity measure for Hybrid SVD and Contourlet. The watermarked image of Contourlet has a PSNR value of $48.9378 \mathrm{~dB}$ and SVD has a PSNR value of 45.1426. After implementing various attacks, Contourlet possess a high PSNR value when compared to DWT. These values are tabulated. The watermark detection using ICA ML extracts the watermark perfectly from the watermarked image. Fig. 15 shows the extracted watermark and Fig. 16-Fig. 18 shows the extracted watermarks from various attacks.

Table 1. PSNR Values for Various Sub-bands and Comparison

\begin{tabular}{|c|c|c|c|c|c|c|}
\hline Sub-bands & $\operatorname{PSNR}(\mathrm{dB})$ & \multirow[t]{2}{*}{ Algorithm } & \multicolumn{4}{|c|}{ PSNR(dB) } \\
\hline 1 & 42.6551 & & $\begin{array}{l}\text { Watermarked } \\
\text { Image }\end{array}$ & $\begin{array}{l}\text { Salt and Pepper } \\
\text { Noise }\end{array}$ & Rotation & $\begin{array}{l}\text { Median } \\
\text { Filtering }\end{array}$ \\
\hline 2 & 41.9311 & Contourlet & 48.9378 & 26.0042 & 28.5734 & 37.0314 \\
\hline 3 & 48.5828 & Hybrid SVD & 45.1426 & 24.3154 & 25.3160 & 35.4531 \\
\hline 4 & 28.4833 & \multirow[t]{2}{*}{ Algorithm } & \multicolumn{4}{|c|}{ Similarity Measure } \\
\hline 5 & 45.9839 & & $\begin{array}{l}\text { Watermarked } \\
\text { Image }\end{array}$ & $\begin{array}{l}\text { Salt and Pepper } \\
\text { Noise }\end{array}$ & Rotation & $\begin{array}{l}\text { Median } \\
\text { Filtering }\end{array}$ \\
\hline 6 & 43.2955 & Contourlet & 0.9616 & 0.9309 & 0.9422 & 0.9455 \\
\hline 7 & 48.9378 & Hybrid SVD & 0.9388 & 0.9301 & 0.9375 & 0.9386 \\
\hline
\end{tabular}

Table 2. Watermark Embedding and Their Extraction Images

\begin{tabular}{|l|l|l|}
\hline \multicolumn{1}{|c|}{ Nature of Image } & \multicolumn{1}{|c|}{ Images } & $\begin{array}{l}\text { Watermarked image from } \\
\text { seventh subband Fig. } 11\end{array}$ \\
\hline Original Image Fig. 6 & & \\
\hline
\end{tabular}




\begin{tabular}{|l|l|l|}
\hline Watermark Fig. 10 & $\begin{array}{l}\text { Extracted watermark from } \\
\text { Rotation Fig. 17 }\end{array}$ \\
\hline $\begin{array}{l}\text { Extracted watermark from salt } \\
\text { and pepper noise Fig. 16 } \\
\text { median filtering Fig. 18 }\end{array}$ &
\end{tabular}

\section{Conclusion}

Performance analysis of digital water marking using contourlet transform are examined and their extraction using ICA ML is attempted in this paper work. From the result it is concluded that contourlet transformation scheme is robust against attacks and also posses considerable PSNR and similarity measure values. Hence Contourlet proves its imperceptibility and robustness and it performs better than Hybrid SVD.

\section{References}

[1] Cox, I., Kilian, J., Leighton, F. T., \& Shamoon, T. (December 1997). Secure spread spectrum watermarking for multimedia. IEEE TRANS, Image Processing, 6, 1673-1689.

[2] Minh, N. D., \& Martin, V. (Dec. 2005). The contourlet transform: An efficient directional multiresolution image representation. IEEE Transaction on Image Processing, 14(12), 2091-2106.

[3] Wang, H. J. M., Su, P. C., \& Kuo, C. C. J. (1998, Dec. 7). Wavelet-based digital image watermarking. Opt. Express, 3(12), 491-496.

[4] Narasimhulu, C. V., \& Prasad, K. S. (2010). A hybrid watermarking scheme using contourlet transform and singular value decomposition. International Journal of Computer Science and Network Security, 10(9).

[5] Xia, X., Boncelet, C. G., \& Arce, G. R. (1997). A multiresolution watermark for digital images. Proceedings of $4^{\text {th }}$ IEEE Int. Conf. Image Processing '97 (pp. 548-551). Santa Barbara, CA.

[6] Kundur, D., \& Hatzinakos, D. (1998). Digital watermarking using multiresolution wavelet decomposition. Proceedings of IEEE Int. Conf. Acoustics, Speech, Signal Processing: Vol. 5 (pp. 2969-2972). Seattle, WA.

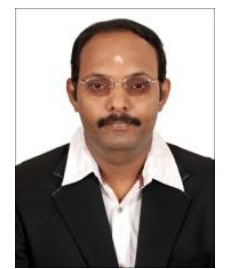

S. Subramanian was born in Trivandrum in 1982. He received his B.E. degree in electronics and instrumentation engineering and M.E. in process control instrumentation from Annamalai University, Annamalai Nagar, Chidambaram, Tamilnadu, India in 2004 and 2012 respectively. Currently he is pursuing the Ph.D. in the field of image processing from Annamalai Univeristy. He is having 10 years of teaching experience in the Department of Instrumentation. His main areas of research interest are control system, process control, image processing.

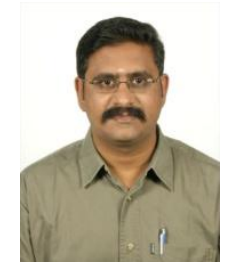

G. Thirugnanam received his B.E, M.E and Ph.D degrees from Annamalai Univesity, Annamalai Nagar, Chidambaram, Tamilnadu, India. Currently he is working as an assistant professor in the Department of Electronics and Instrumentation Engineering, Annamalai University. His area of interest are process control, industrial instrumentation, watermarking, image processing, wavelet transform. 